\title{
ASR of mortars containing glass
}

\section{Serpa D. ${ }^{1}$, Santos Silva A. ${ }^{2}$ J. de Brito ${ }^{3}$, Pontes J. ${ }^{4}$ and Soares D. ${ }^{5}$}

${ }^{1}$ DECivil-IST, Technical University of Lisbon, Av. Rovisco Pais, 1049-001, Lisbon, Portugal, diogo_serpa@hotmail.com

${ }^{2}$ National Laboratory of Civil Engineering, Av. do Brasil 101, 1700-066, Lisbon, Portugal, ssilva@lnec.pt

${ }^{3}$ Full Professor, Department of Civil Engineering, Architecture and Georresources, IST - Technical University of Lisbon, Av. Rovisco Pais, 1049-001, Lisbon, Portugal, e-mail: jb@ civil.ist.utl.pt, Phone: (351) 218419709; Fax: (351) 21 8497650), Corresponding author

${ }^{4}$ DECivil-IST, Technical University of Lisbon, Av. Rovisco Pais, 1049-001, Lisbon, Portugal, jorgempontes@gmail.com

${ }^{5}$ National Laboratory of Civil Engineering, Av. do Brasil 101, 1700-066, Lisbon, Portugal, dsoares@lnec.pt

\begin{abstract}
Glass is a material that raises environmental issues in terms of both recycling and dumping. Some studies have shown the potential of glass as pozzolan when used as fine powder. Its use as aggregate in cementitious materials may in certain circumstances lead to alkali silica reaction (ASR) related problems.
\end{abstract}

ASR is one of the most studied deleterious degradation mechanisms of concrete, which is particularly harmful. Once detected in a concrete structure, ASR is very difficult to stop. It is nowadays possible to use mineral additions like natural pozzolans or subproducts with pozzolanic reactivity to inhibit ASR in new concrete.

This work shows the results of using a Portuguese recycled glass material as aggregate or as pozzolan in cement based mortars, to ascertain their applicability in concrete. The incorporation of glass as cement replacement or even as aggregate can decrease the ASR effects and its efficiency is related with the replacement ratio.

Keywords: Alkali-silica reaction, Construction and demolition waste, Glass, Recycling 